\title{
ANÁLISE DA PRODUÇÃO CIENTÍFICA FONOAUDIOLÓGICA NACIONAL SOBRE GAGUEIRA
}

\author{
Analysis of speech and language therapy national \\ scientific production on stuttering
}

Wladimir Alberti Pascoal de Lima Damasceno (1), Silvia Friedman (2)

\begin{abstract}
RESUMO
Objetivo: analisar a produção científica fonoaudiológica nacional no que diz respeito à gagueira, entre os anos de 1980 a 2008, considerando: período da publicação; distribuição de frequência por período; tipos de publicação; temáticas abordadas; vertente epistemológica e área da Fonoaudiologia a que o trabalho pertence. Métodos: seleção e análise de publicações em livros, capítulos de livros e periódicos nacionais de Fonoaudiologia. Resultados: foram encontradas 131 publicações: estando 0,8\% na década de $80 ; 15,2 \%$ na de 90 ; e $84 \%$ no período de $2000-2008$. Quanto ao tipo formam publicados, $8,4 \%$ em livros, $42 \%$ de capítulos de livro e $49,6 \%$ de periódicos. Quanto às temáticas, foram mais frequentes: "Clínica Fonoaudiológica" (50\%); "Reflexões Teóricas" (9,4\%); "Características da Fluência" (7,2\%); "Gagueira e Audição" (5,8\%); "Atitudes em Relação à Gagueira" (5,2\%); "Origem da Gagueira" $(5,2 \%)$ e "Características da Gagueira" (4,4\%). Quanto à vertente epistemológica, $74 \%$ era positivista, $22,2 \%$ dialético histórica e 3,8\% fenomenológica. As áreas fonoaudiológicas mais encontradas foram: Motricidade Orofacial $(37,4 \%)$, Linguagem (34,3\%) e Nenhuma das Áreas Fonoaudiológicas (25,2\%). Conclusão: a produção pesquisada teve o crescimento mais expressivo de 2000 a 2008. O fato de a produção encontrar-se principalmente na forma de artigos e capítulos de livros revela interesse tanto em pesquisa como na construção de teoria. A presença das três vertentes epistemológicas indica que o tema gagueira é controverso e complexo, isso é indicado também pelo fato de a produção distribuirse pelas áreas de Motricidade Orofacial e Linguagem. Há necessidade de desenvolver a produção na área da Saúde Pública.
\end{abstract}

DESCRITORES: Gagueira; Fonoaudiologia; Indicadores de Produção Científica

\section{INTRODUÇÃO}

A controvérsia a respeito da gagueira, no campo científico, motivou o interesse em conhecer as tendências e características da produção científica fonoaudiológica nacional sobre essa temática.

Pesquisar a produção científica de uma área é considerado importante porque ao se delinear seu panorama histórico, permite-se conhecer as tendências de investigação e se oferecem informações

(1) Aluno do curso de Graduação em Fonoaudiologia da Universidade Potiguar, UnP, Natal, RN.

(2) Fonoaudióloga; Docente Titular do Curso de Fonoaudiologia da Pontifícia Universidade Católica de São Paulo, PUC-SP, São Paulo, SP; Doutora em Psicologia Social pela Pontifícia Universidade Católica.

Conflito de interesses: inexistente indispensáveis para sua execução futura ${ }^{1,2}$. Tais pesquisas favorecerem o crescimento da produção, bem como aperfeiçoam publicações subsequentes ${ }^{3-6}$. Além disso, a indicação dos direcionamentos seguidos por uma área permite elaborar uma crítica interna sobre a produção, o que é considerado condição favorável para que o conhecimento incorporado por uma comunidade possa cumprir seu papel transformador ${ }^{7-9}$.

Entre os veículos de divulgação do conhecimento na comunidade científica, os periódicos assumem lugar de destaque ${ }^{10-12}$. São valorizados por terem avaliação de corpo editorial e/ou consultores ad hoc, o que favorece a qualidade da produção e cria critérios para norteá-la ${ }^{13}$. Essa avaliação é considerada importante, por serem estes instrumentos de disseminação e acessibilidade ao conhecimento e por permitirem medir o avanço e o rigor científico ${ }^{14}$. 
O volume de artigos publicados em periódicos indexados em bases de dados é um dos principais parâmetros indicativos do vigor científico ${ }^{9}$.

Além dos periódicos, nos campos do saber relativos às denominadas soft sciences, como é o caso dos campos da Fonoaudiologia que tem como objeto de estudo e intervenção a linguagem, e dentro dela a fluência de fala e seus problemas, merece igualmente atenção a produção cientifica publicada em livros e capítulos de livros ${ }^{6}$, porque estes também permitem compreender quantitativa e qualitativamente características e tendências dessa produção.

Estudos sobre a produção do conhecimento, na área dos Distúrbios da Comunicação, enfatizam a necessidade de se refletir sobre as temáticas e os aspectos teórico-metodológicos que caracterizam a produção bibliográfica. Isso é proposto como condição para identificar os domínios de investigação historicamente priorizados e estabelecer ações e campos estratégicos para as pesquisas futuras $3,6,9,11$.

Diante disso, o objetivo do presente estudo é realizar o levantamento e a caracterização da produção científica fonoaudiológica brasileira acerca de gagueira, entre os anos de 1980 e 2008, considerando: período da publicação, distribuição de frequência por período, tipos de publicação, temáticas abordadas, vertente epistemológica dos trabalhos e área da Fonoaudiologia a que o trabalho pertence.

\section{MÉTODOS}

A Pesquisa desenvolveu-se no Núcleo de Estudos e Pesquisa em Fluência de Fala - NEPFF vinculado à Linha de Pesquisa Linguagem e Subjetividade do Programa de Estudos Pós-Graduados em Fonoaudiologia da Pontifícia Universidade Católica de São Paulo - PUCSP e ao Instituto CEFAC - Ação Social em Saúde e Educação.

Os tipos de publicações analisadas foram os livros, capítulos de livros e periódicos nacionais especializados em Fonoaudiologia. Os periódicos analisados foram: Revista da Sociedade Brasileira de Fonoaudiologia; Revista Distúrbios da Comunicação; Revista CEFAC - Atualização Científica em Fonoaudiologia e Educação; Pró-Fono Revista de Atualização Científica; Revista Fono Atual e Jornal Brasileiro de Fonoaudiologia. Nesses, pelo menos um dos autores era fonoaudiólogo.

O levantamento teve início em 1980, por registrar a primeira obra brasileira sobre 0 assunto, 0 livro "Gagueira: Teoria e Tratamento de Adultos e Crianças", de Jakubovicz ${ }^{15}$.
O acesso aos periódicos, livros e capítulos de livro foi manual na biblioteca da Universidade Potiguar - UnP - Campus Salgado Filho e via internet nas bases de dados LILACS e MEDLINE. Utilizaram-se as palavras-chaves: gagueira, fluência $e$ disfluência. Uma análise prévia dos artigos, livros e capítulos de livros permitiu selecionar os documentos que tratavam de gagueira.

Organizou-se a análise das publicações a partir da distribuição de sua frequência, considerando o período das publicações, o tipo de publicação (livro, capítulo, artigo), a temática abordada, a vertente epistemológica de filiação e a área fonoaudiológica a que pertence.

Para compreender as características teóricoconceituais e caracterizar a vertente epistemológica, leram-se os trabalhos na íntegra. Formam tomadas com base para essa classificação as três vertentes definidas no campo epistemológico como constitutivas da ciência desde o final do século XIX, a saber: o positivismo, a fenomenologia e a dialética-histórica ${ }^{16}$. A primeira se refere a uma visão centrada no organismo e nos sintomas de fala, a segunda se refere a aquilo que através da percepção o pesquisador pode apreender de seu objeto e a terceira se refere a uma visão centrada no sujeito, sua subjetividade e sua história ${ }^{17}$.

As temáticas geralmente evidenciavam-se no título do trabalho. A leitura integral do texto ajudou a corroborar a pertinência da definição temática. Esta obedeceu ao critério da abrangência para permitir congregar maior número de trabalhos em cada uma.

Para as áreas de atuação fonoaudiológica, com base no Conselho Federal de Fonoaudiologia consideraram-se as seguintes: Linguagem, Audiologia, Motricidade Orofacial, Voz e Saúde Coletiva ${ }^{18}$. Essa classificação permite respostas múltiplas.

Para a análise estatística dos dados utilizou-se o teste Qui-Quadrado $\left(\mathrm{X}^{2}\right)$, tomando 5\% como nível de significância. Os resultados obtidos $\left(X^{2}\right.$, graus de liberdade e p-value) são apresentados ao final das tabelas.

\section{RESULTADOS}

O total da produção encontrada foi de 131 documentos.

A Tabela 1 mostra a distribuição desses 131, segundo o tipo de publicação (artigos, capítulos de livros e livros), da produção científica fonoaudiológica nacional sobre gagueira de acordo com o tempo e a temática.

Quanto ao tempo, o crescimento mais significante foi de artigos e capítulos de livros. O teste 
Tabela 1 - Distribuição, segundo tipo de publicação, da produção científica fonoaudiológica nacional sobre gagueira de acordo com o tempo e com a temática

\begin{tabular}{|c|c|c|c|c|c|c|c|c|}
\hline \multirow{2}{*}{$\begin{array}{l}\text { Produção por Período } \\
\text { de } 10 \text { anos }\end{array}$} & \multicolumn{2}{|c|}{ Livro } & \multicolumn{2}{|c|}{$\begin{array}{l}\text { Capítulos de } \\
\text { Livros }\end{array}$} & \multicolumn{2}{|c|}{$\begin{array}{l}\text { Artigos em } \\
\text { Periódicos }\end{array}$} & \multicolumn{2}{|c|}{ População } \\
\hline & $\mathbf{F}$ & $\%$ & $\mathbf{F}$ & $\%$ & $\mathbf{F}$ & $\%$ & $\mathbf{F}$ & $\%$ \\
\hline 1980 a 1989 & 1 & 9,10 & 0 & 0 & 0 & 0 & 1 & 0,76 \\
\hline 1990 a 1999 & 5 & 45,5 & 5 & 9,10 & 10 & 15,4 & 20 & 15,3 \\
\hline 2000 a 2008 & 5 & 45,5 & 50 & 90,9 & 55 & 84,6 & 110 & 84,0 \\
\hline Total para a categoria & 11 & 100 & 55 & 100 & 65 & 100 & 131 & 100 \\
\hline \multicolumn{9}{|l|}{$\begin{array}{c}\text { Temáticas mais } \\
\text { frequentes relacionadas } \\
\text { à gagueira }\end{array}$} \\
\hline Clínica Fonoaudiológica & 08 & 61,5 & 44 & 77 & 17 & 33,3 & 69 & 57,5 \\
\hline Reflexões Teóricas & 02 & 15,4 & 06 & 10,5 & 05 & 9,8 & 13 & 10,8 \\
\hline Característica da Fluência & 00 & 00 & 02 & 3,5 & 08 & 15,7 & 10 & 8,3 \\
\hline Gagueira e Audição & 00 & 00 & 02 & 3,5 & 06 & 11,8 & 08 & 6,8 \\
\hline $\begin{array}{l}\text { Atitudes em Relação à } \\
\text { Gagueira }\end{array}$ & 01 & 7,7 & 00 & 00 & 06 & 11,8 & 07 & 5,8 \\
\hline Origem da Gagueira & 01 & 7,7 & 01 & 2 & 05 & 9,8 & 07 & 5,8 \\
\hline Característica da Gagueira & 01 & 7,7 & 02 & 3,5 & 03 & 7,8 & 06 & 5,0 \\
\hline Total para a categoria & 13 & 100 & 57 & 100 & 50 & 100 & 120 & 100 \\
\hline \multicolumn{2}{|c|}{$\begin{array}{c}\text { Resultados do } \mathrm{X}^{2} \text { (Tempo) } \\
\text { Resultados do } \mathrm{X}^{2} \text { (Temáticas) }\end{array}$} & $\begin{array}{l}X^{2}=21,1 \\
X^{2}=29,4\end{array}$ & \multicolumn{4}{|c|}{ Graus de liberdade $=4$} & \multicolumn{2}{|c|}{$\begin{array}{l}p \text {-value }=0,0003 \\
p \text {-value }=0,003\end{array}$} \\
\hline
\end{tabular}

qui-quadrado indicou que de forma geral o aumento do número de publicações durante o período de 1980 a 2008 foi significante $\left(P\left(X^{2}>21,1\right)=0,0003\right)$. Com contribuições semelhantes, esse aumento resulta principalmente de publicações na forma de Capítulos de Livros e de Artigos em Periódicos, com a publicação de Livros tendo menor importância (Capítulos de Livros - Artigos em Periódicos: $P\left(X^{2}>1.08\right)=0.30$; Livros - Capítulos de Livros: $\mathrm{P}\left(\mathrm{X}^{2}>15.27\right)=5 \times 10^{-4}$; Livros - Artigos em Periódicos: $\left.P\left(X^{2}>12.05\right)=2 \times 10^{-3}\right)$.

Quanto às temáticas, as sete mais frequentes foram: Clínica Fonaudiológica, Reflexões Teóricas, Característica da Fluência, Gagueira e Audição, Atitudes em Relação à Gagueira, Origem da Gagueira e Características da Gagueira perfazendo um total de 120 documentos, sendo que os outros 11 tinham cada um uma temática diferente e, por isso, optamos por não incluí-los na análise subsequente. A temática Clínica Fonoaudiológica, a mais frequente de todas, corresponde a $57,5 \%$ do total de publicações consideradas, enquanto as demais seis temáticas, com contribuições estatísticas semelhantes, correspondem aos $42,5 \%$ restantes.

A Tabela 2 mostra a distribuição das temáticas pelas vertentes epistemológicas $O$ teste Qui-Quadrado mostrou que a preponderância da vertente positivista, em número absoluto, não tem significância estatística.

A Tabela 3 mostra a distribuição temática pelas áreas de atuação fonoaudiológica, sendo que cinco trabalhos pertenceram a mais de uma área. Testes Qui-Quadrado par a par das áreas de atuação mostraram que as produções nas áreas da Linguagem e Motricidade Orofacial foram significativamente superiores às demais (Linguagem-Audição: $\mathrm{P}\left(\mathrm{X}^{2}>38,7\right)<5 \times 10^{-5}$; Linguagem - Nenhuma das áreas Fonoaudiológicas: $\mathrm{P}\left(\mathrm{X}^{2}>31,8\right)<5 \times 10^{-5}$; Motricidade Orofacial - Audição: $\mathrm{P}\left(\mathrm{X}^{2}>50,0\right)<5 \times 10^{-5} \mathrm{e}$ Motricidade Orofacial - Nenhuma das áreas Fonoaudiológicas: $\left.\mathrm{P}\left(\mathrm{X}^{2}>41,1\right)<5 \times 10^{-5}\right)$. Por sua vez, as contribuições das produções nas áreas da Linguagem e Motricidade Orofacial foram de relevância semelhante (Linguagem - Motricidade Orofacial: $\left.P\left(X^{2}>5.42\right)=0.49\right)$ e a área da audição foi, dentre todas, a de menor importância (Audição - Nenhuma das áreas Fonoaudiológicas: $\left.P\left(X^{2}>26.36\right)<0.0001\right)$.

\section{DISCUSSÃO}

Somente no século XXI houve crescimento significante da produção sobre gagueira. Nos primeiros oito anos deste século a produção elevou-se a um 
Tabela 2 - Distribuição temática segundo a vertente epistemológica

\begin{tabular}{|c|c|c|c|c|c|c|c|c|}
\hline \multirow{2}{*}{$\begin{array}{c}\text { Temáticas mais } \\
\text { frequentes relacionadas } \\
\text { à gagueira }\end{array}$} & \multicolumn{2}{|c|}{$\begin{array}{l}\text { Positivista } \\
\text { (P) }\end{array}$} & \multicolumn{2}{|c|}{$\begin{array}{c}\text { Dialético- } \\
\text { Histórica (DH) }\end{array}$} & \multicolumn{2}{|c|}{$\begin{array}{c}\text { Fenomenológica } \\
\text { (FEN) }\end{array}$} & \multicolumn{2}{|c|}{ População } \\
\hline & $\mathbf{F}$ & $\%$ & $\mathbf{F}$ & $\%$ & $\mathbf{F}$ & $\%$ & $\mathbf{F}$ & $\%$ \\
\hline Clínica Fonoaudiológica & 44 & 51,1 & 21 & 72,4 & 04 & 80,0 & 69 & 57,5 \\
\hline Reflexões Teóricas & 08 & 9,30 & 04 & 13,8 & 01 & 20,0 & 13 & 10,8 \\
\hline $\begin{array}{l}\text { Características da } \\
\text { Fluência }\end{array}$ & 09 & 10,5 & 01 & 3,45 & 00 & 00 & 10 & 8,33 \\
\hline Gagueira e Audição & 08 & 9,30 & 00 & 00 & 00 & 00 & 08 & 6,67 \\
\hline $\begin{array}{c}\text { Atitudes em Relação à } \\
\text { Gagueira }\end{array}$ & 06 & 6,98 & 01 & 3,45 & 00 & 00 & 07 & 5,83 \\
\hline Origem da Gagueira & 06 & 6,98 & 01 & 3,45 & 00 & 00 & 07 & 5,83 \\
\hline $\begin{array}{c}\text { Características da } \\
\text { Gagueira }\end{array}$ & 05 & 5,81 & 01 & 3,45 & 00 & 00 & 06 & 5,00 \\
\hline Total para a categoria & 86 & 100 & 29 & 100 & 05 & 100 & 120 & 100 \\
\hline Resultados do $\mathrm{X}^{2}$ & \multicolumn{2}{|c|}{$X^{2}=9,89$} & \multicolumn{4}{|c|}{ Graus de liberdade $=12$} & -valu & \\
\hline
\end{tabular}

Tabela 3 - Distribuição de temáticas por áreas de atuação fonoaudiológica

\begin{tabular}{|c|c|c|c|c|c|c|c|c|c|c|}
\hline \multirow{2}{*}{$\begin{array}{c}\text { Temáticas mais } \\
\text { frequentes } \\
\text { relacionadas à } \\
\text { gagueira }\end{array}$} & \multicolumn{2}{|c|}{ Linguagem } & \multicolumn{2}{|c|}{$\begin{array}{l}\text { Motricidade } \\
\text { Orofacial }\end{array}$} & \multicolumn{2}{|c|}{ Audição } & \multicolumn{2}{|c|}{$\begin{array}{c}\text { Nenhuma } \\
\text { das Áreas } \\
\text { Fonoaudiológicas }\end{array}$} & \multicolumn{2}{|c|}{ População } \\
\hline & $F$ & $\%$ & $\mathbf{F}$ & $\%$ & $\mathbf{F}$ & $\%$ & $F$ & $\%$ & $\mathbf{F}$ & $\%$ \\
\hline $\begin{array}{c}\text { Clínica } \\
\text { Fonoaudiológica }\end{array}$ & 32 & 74,4 & 31 & 64,6 & 00 & 00 & 06 & 23,1 & 69 & 55,2 \\
\hline $\begin{array}{l}\text { Reflexões } \\
\text { Teóricas }\end{array}$ & 01 & 2,33 & 01 & 2,08 & 00 & 00 & 11 & 42,3 & 13 & 10,4 \\
\hline $\begin{array}{l}\text { Características } \\
\text { da Fluência }\end{array}$ & 03 & 6,98 & 09 & 18,8 & 00 & 00 & 00 & 00 & 12 & 9,60 \\
\hline $\begin{array}{l}\text { Gagueira e } \\
\text { Audição }\end{array}$ & 01 & 2,33 & 00 & 00 & 07 & 87,5 & 01 & 3,85 & 9 & 7,20 \\
\hline \multicolumn{11}{|l|}{ Atitudes em } \\
\hline $\begin{array}{l}\text { Relação à } \\
\text { Gagueira }\end{array}$ & 01 & 2,33 & 00 & 00 & 00 & 00 & 06 & 23,1 & 7 & 5,60 \\
\hline $\begin{array}{l}\text { Origem da } \\
\text { Gagueira }\end{array}$ & 03 & 6,98 & 03 & 6,25 & 01 & 12,5 & 01 & 3,85 & 8 & 6,40 \\
\hline $\begin{array}{l}\text { Características } \\
\text { da Gagueira }\end{array}$ & 02 & 4,65 & 04 & 8,33 & 00 & 00 & 01 & 3,85 & 7 & 5,60 \\
\hline $\begin{array}{c}\text { Total para a } \\
\text { categoria }\end{array}$ & 43 & 100 & 48 & 100 & 08 & 100 & 26 & 100 & 125 & 100 \\
\hline \multicolumn{3}{|c|}{ Resultados do $\mathrm{X}^{2}$} & $x^{2}=15$ & & Gras & e liber & lade $=18$ & & ue $<0$, & \\
\hline
\end{tabular}

número cinco vezes superior à somatória das duas décadas anteriores. Tais dados demonstram que o crescimento da produção acompanha a trajetória identificada em outras áreas do campo fonoaudiológico nacional $6,7,19$.

Tal crescimento relaciona-se ao aumento do número de profissionais graduados em Fonoaudiologia; aumento da demanda por docentes para atuar em novas instituições de ensino; investimento dessas instituições na qualificação de seu corpo docente; exigências da Coordenação de Aperfeiçoamento do Pessoal de Nível Superior (CAPES) e crescimento do número de fonoaudiólogos inseridos em Programas Pós-Graduados (PGs) em nível de Mestrado e Doutorado ${ }^{6}$.

A preponderância de artigos em periódicos científicos indexados, na produção aqui estudada, por favorecer a velocidade, acessibilidade e visibilidade 
da produção de conhecimento ${ }^{3}$, tende a incrementar novas publicações, à medida que favorece o intercâmbio e a divulgação dos trabalhos científicos produzidos numa área, impulsiona novas pesquisas e alimenta o desenvolvimento de linhas de pesquisa ${ }^{6}$.

A preponderância dos artigos, ainda, remete ao fato de que tais veículos são sempre relatos bastante sintéticos do resultado de pesquisas, restritos à compreensão de especialistas. Essa compreensão é possível, porque todos os conceitos e teorias que os alimentam estão assentados e são aceitos pelos profissionais que compõem uma área ${ }^{18}$. Dessa definição se pode depreender que sobre a questão gagueira, há uma comunidade científica positivista, cujos estudos estão centrados no organismo e nos sintomas de fala e uma dialético-histórica, cujos estudos estão centrados no sujeito falante face sua subjetividade e sua história ${ }^{21}$.

A marcada presença de capítulos de livros na produção estudada remete ao fato de que tais veículos se prestam à construção de teorias e conceitos que explicam e defendem pontos de vista diferentes e, até, antagônicos, típicos da falta de consenso em torno de um paradigma ${ }^{20}$. Isso faz sentido com o fato de que os capítulos foram o principal veículo de publicação na temática mais frequente e que esta apresentou trabalhos nas três vertentes epistemológicas ou paradigmas aqui encontrados.

Outros estudos sobre a produção científica fonoaudiológica brasileira também mostraram a presença de mais de um paradigma na produção ${ }^{6}$. Embora sem significância estatística, a preponderância, em números absolutos, da vertente positivista na produção estudada, confirma outras investigações que definem ser o positivismo a epistemologia hegemônica dentro da área da saúde ${ }^{22}$, área dentro da qual a Fonoaudiologia como um todo está atualmente classificada no campo da ciência.

A distribuição dos trabalhos da temática "Clínica Fonoaudiológica", por duas áreas de atuação fonoaudiológica, Motricidade Orofacial e Linguagem, juntamente com a presença dos três paradigmas na produção estudada, refletem a complexidade do objeto gagueira, bem como a falta de consenso sobre sua natureza ${ }^{21}$. Além disso, o fato de ser "Clínica Fonoaudiológica" a temática mais abordada, pode ser sugestivo do envolvimento do fonoaudiólogo brasileiro no estudo e na busca de maior compreensão de questões que abrangem o tratamento da gagueira.

Classificar "Reflexões Teóricas" preferencialmente em Nenhuma das Áreas Fonoaudiológicas representa o fato de a reflexão teórica não pertencer ao campo específico da Fonoaudiologia e sim, ao da Filosofia, o qual perpassa todas as áreas de conhecimento. A necessidade de refletir ou filosofar sobre gagueira, também remete à complexidade e à falta de consenso sobre esse objeto.

A área de Motricidade Orofacial mostrou produção coesa em torno da vertente positivista. A área de Linguagem, em que aparecem todas as vertentes, mostra-se como o espaço dos antagonismos epistemológicos.

\section{CONCLUSÃO}

O levantamento e a caracterização da produção científica fonoaudiológica nacional no que diz respeito à gagueira, entre os anos de 1980 a 2008, mostrou que o crescimento mais expressivo ocorreu nos primeiros oito anos do presente século.

Os resultados desse estudo mostraram que a produção científica tem demandado tanto pesquisas, na forma de artigos, quanto à elaboração teórica, na forma de capítulos de livros. Considerando ser "Clínica Fonoaudiológica" a temática mais abordada tanto nos artigos como nos capítulos de livros, conclui-se que a abordagem clínica da gagueira é a questão que mais tem ocupado os fonoaudiólogos.

O fato de a produção científica fonoaudiológica nacional sobre gagueira apresentar trabalhos nas três vertentes epistemológicas conhecidas, sugere tanto a existência de controvérsias nas concepções que sustentam a questão, como a sua complexidade. Estas também se expressaram no fato de os trabalhos estarem preponderantemente distribuídos entre as áreas de Motricidade Orofacial e de Linguagem, que tomam o objeto gagueira de formas bastante distintas.

A preponderância da vertente positivista na produção dos trabalhos sobre gagueira indica que os estudos têm explicado o fenômeno mais do ponto de vista do organismo que do sujeito.

A distribuição das temáticas encontradas pelas áreas de especialidade da Fonoaudiologia indica a falta de pesquisas na área de Saúde Pública. 


\section{ABSTRACT}

Purpose: to analyze the Brazilian Speech-Language and Hearing Sciences production on stuttering, between the years 1980 and 2008, considering the: publication period; distribution per period; types of publication; themes; epistemological filiations and the area of Speech-Language and Hearing Sciences. Methods: selection and analysis of books, book chapters, and scientific articles published in national journals of Speech-Language and Hearing Sciences. Results: 131 publications were found: $0.8 \%$ published during the $80 \mathrm{~s} ; 15.2 \%$ during the $90 \mathrm{~s}$; and $84 \%$ from 2000 to 2008 . Regarding the type of publications, $8.4 \%$ were books; $42 \%$ book chapters, and $49,6 \%$ research articles. Regarding the themes, the most frequent ones were: Speech-Language and Hearing Clinic (50\%); Theoretical Reflections (9.4\%); Fluency Characteristics (7.2\%); Stuttering and Hearing (5.8\%); Attitudes towards Stuttering (5.2\%); Origin of Stuttering (5.2\%); and Characteristics of Stuttering (4.4\%). Regarding the epistemological feature: $74 \%$ were positivist; $22.2 \%$ dialectical historical; $3.8 \%$ phenomenological. The Speech-Language and Hearing areas most present in the written production were Oral Sensorial Motor System (37.4\%), Language (34.3\%) and None of the Speech-Language (25.2\%). Conclusion: the scientific production on stuttering had the most expressive increase during 2000 to 2008. The appearance of the production mainly as scientific articles and book chapters reveals an interest both in research and in theoretical construction. The presence of the three epistemological filiations suggests the controversy and complexity of the stuttering issue, this is also suggested by the fact that the production is predominantly distributed by the Oral Sensorial Motor System and Language areas. It is needed to develop the production in the Public Health area.

KEYWORDS: Stuttering; Speech, Language and Hearing Sciences; Scientific Publication Indicators

\section{REFERÊNCIAS}

1. Berberian APA, Krüguer $S$, Guarinello AC, Massi GA de A. A produção do conhecimento em Fonoaudiologia em comunicação suplementar e/ ou alternativa: análise de periódicos. Rev. CEFAC. 2009; 11(2):258-66.

2. Castro MLS. Educação e Economia: análise de artigos publicados em periódicos brasileiros: 19822000. Análise. 2006; 17(2):224-33.

3. Campanatti-Ostiz H, Andrade CRF. Periódicos nacionais em fonoaudiologia: caracterização estrutural. Rev Soc Bras Fonoaudiol. 2005; 10(3):147-54.

4. Campanatti-Ostiz H, Andrade CRF. Periódicos nacionais em fonoaudiologia: caracterização de termos. Rev Fonoaudiol Bras. 2005; 3(1):1-4.

5. Freire RM, Passos MC. Uma análise da produção de conhecimento no interior do P.E.P.G. em Fonoaudiologia: de sua fundação até o milênio. Rev Dist Comun. 2005; 17(1):37-43.

6. Munhoz CMA, Massi G, Berberian AP, Giroto CRM, Guarinello AC. Análise da produção científica nacional fonoaudiológica acerca da linguagem escrita. Pró-Fono. 2007; 19(3):249-58.

7. Berberian APA, Ferreira LP, Corteletti LCBJ, Azevedo JBM de, Marques JM. A produção do conhecimento em distúrbios da comunicação: análise de periódicos (2000 a 2005). Rev Soc Bras Fonoaudiol. 2009; 14(2):153-9

8. Yamamoto $\mathrm{OH}$, Menadro PRM, Koller $\mathrm{SH}$, Lobianco AC, Hutz CS, Bueno JL, Guedes MC. Avaliação de periódicos científicos brasileiros da área da psicologia. Cienc Inf. 2002; 31(2):163-77.

9. Ferreira LP, Russo ICP. Fonoaudiólogos doutores no Brasil: análise das teses segundo áreas de atuação e programas. Pró-Fono. 2004; 16(1):119-30.

10. Bufrem LS, Sorribas TV. Produção científica em Ciência da Informação: análise temática em artigos de revistas brasileiras. Persp Ciênc Inf. 2007; 12(1):38-49.

11. Campanatti-Ostiz H, Andrade CRF. Periódicos nacionais em fonoaudiologia: caracterização de indicador de impacto. Pró-Fono. 2006; 18(1):99-110. 12. Freitas $C M$ de. $A$ produção científica sobre 0 ambiente na saúde coletiva. Cad Saúde Pública. 2005; 21(3):679-701.

13. Ferreira NS, Yoshida EMP. Produção científica sobre psicoterapias breves no Brasil e demais países latinoamericanos (1990-2000). Est. Psicol. 2004; 3(3):523-31.

14. Romancini R. Periódicos brasileiros em comunicação: histórico e análise preliminar. Verso e Reverso. 2004; 2(39):1-18.

15. Jakubovicz R. Gagueira: teoria e tratamento de adultos e crianças. Rio de Janeiro: Antares, 1980. 
16. Spink MJP. Caminando sobre huevos: una reflexión construccionista sobre la investigación. Athenea Digital. 2006; 9(1),168-83.

17. Gil AC. Métodos e técnicas em pesquisa social. 6. ed. São Paulo: Atlas, 2008.

18. Conselho Federal de Fonoaudiologia [Internet]. RESOLUÇÃO CFFa no 320, de 17 de fevereiro de 2006 [Acesso em 25 Out 2009] Disponível em: http://www.fonoaudiologia.org.br/servlet/ConsultaL egislacao ?acao=V\&leild $=35$.

19. Vasconcelos SV de, Pessoa ACRG, Farias AP de S. Caracterização das publicações periódicas em Fonoaudiologia e Neurociências: estudo sobre os tipos e temas de artigos e visibilidade na área da linguagem. Rev CEFAC. 2009; 11(1):50-8.

20. Kuhn TS. A estrutura das revoluções científicas. 6. ed. São Paulo: Perspectiva; 2001.

21. Oliveira OS, Friedman S. Clínica da Gagueira: diferentes paradigmas e suas consequências. In: RHF David, Barbosa OS. Cadernos da Fonoaudiálogo - série linguagem - vol. 1. São Paulo: Lovise; 2006. p.7-13.

22. Tesser CD, Luz MT. Uma introdução às contribuições da epistemologia contemporânea para a medicina. Ciênc. Saúde Coletiva. 2002; 7(2):363-72.

DOI: 10.1590/S1516-18462010005000131

RECEBIDO EM: 15/11/2009

ACEITO EM: 18/03/2010

Endereço para correspondência:

Wladimir Alberti Pascoal de Lima Damasceno

Travessa Progresso, 02

Natal - RN

CEP: 59020-565

E-mail: wladimirdamasceno@gmail.com 